Ágora Rev Cient. 2015; 02(2): 143 -144

\title{
Medicina tradicional y complementaria en el marco de las prestaciones de servicios de salud.
}

La Medicina Tradicional y Complementaria, es un componente muy importante de las prestaciones de servicios de salud en el Perú y en el Mundo, se practica cotidianamente y la demanda de estos servicios va en aumento. La medicina tradicional de calidad, seguridad y eficacia comprobadas contribuye a asegurar el acceso de todas las personas a la atención de salud. La medicina tradicional y natural considera al hombre de manera holística; es decir, en su totalidad y dentro de un aspecto ecológico, y parte de que la falta de salud o la enfermedad proceden de un desequilibrio del hombre en su sistema ecológico total y no sólo del agente causal y la evolución patógena. Esta terapéutica constituye un medio más seguro de promoción de la medicina tradicional y natural; de ahí la importancia de la interacción en la atención primaria de salud.

Una mayor y mejor asistencia sanitaria no consisten, tan sólo, en la práctica de costosos tratamientos y sofisticados equipos vinculados a la biotecnología, sino ante todo, en educar sanitariamente a la población en la utilización de los recursos de la naturaleza como son, el uso de las plantas, agua, luz, aire, sol, arcilla y alimentos; así como, el empleo de técnicas fisioterapéuticas y la búsqueda de terapias menos agresivas y sin efectos secundarios.

Para mantener una adecuada salud mental y física debe existir un equilibrio biológico. Cuando éste se rompe aparece la enfermedad. Muchos países reconocen actualmente la necesidad de elaborar un enfoque coherente e integral de la atención de salud, que facilite a los profesionales de la salud y, muy especialmente, a los usuarios de los servicios de salud, el acceso a la Medicina Tradicional de manera segura, respetuosa, asequible y efectiva. En todo el mundo, la medicina tradicional es el pilar principal o el complemento de la prestación de servicios de salud. La estrategia de la OMS sobre medicina tradicional 2014-2023 vuelve a evaluar y desarrollar la estrategia de la OMS sobre medicina tradicional 2002-2005, y señala el rumbo de la medicina tradicional y complementaria (MTC) para el próximo decenio $(1,2)$.

La MTC es una parte importante y con frecuencia subestimada de la atención de salud. Se la practica en casi todos los países del mundo, y la demanda va en aumento.Una estrategia mundial destinada a promover la integración, reglamentación y supervisión apropiadas de la MTC será de utilidad para los países que desean desarrollar políticas dinámicas relativas a esta parte importante, y con frecuencia vigorosa y expansiva, de la atención de salud. Frecuentemente, las necesidades individuales mueven a las personas a recurrir a la MTC $(3,4)$. Algunos estudios revelan que los pacientes que sufren determinados trastornos crónicos utilizan servicios de MTC con mayor frecuencia. Por ejemplo, un estudio realizado en los Estados Unidos indicó que los pacientes con enfermedades reumáticas que consultaban a médicos osteópatas en el contexto de la medicina de familia representaban el 23\% de las visitas en un período de un año (5). En Francia, los pacientes con trastornos crónicos del aparato locomotor representan una gran parte de las consultas a médicos que ofrecen tratamientos alternativos a la medicina convencional (6). Un amplio número de pacientes con esclerosis múltiple recurren a tratamientos de medicinas complementarias y alternativas: la prevalencia del uso va del $41 \%$ en España, al $70 \%$ en el Canadá y el 82\% en Australia (7). En China, según datos de los servicios nacionales de seguimiento de la medicina tradicional china, las cinco enfermedades principales que motivaron admisiones en hospitales de medicina tradicional china en 2008 fueron: accidente cerebrovascular, hernia discal, hemorroides, cardiopatía isquémica e hipertensión esencial (8). La República de Corea notificó que en 2011 las principales enfermedades que motivaron admisiones en hospitales de medicina tradicional coreana fueron: trastornos del sistema articular y muscular, dispepsia, artrosis de rodilla y trastornos del nervio facial.

En este contexto debemos de manifestar que hay un creciente interés por la Medicina Tradicional y Complementaria, sin embargo aún persisten muchos interrogantes acerca de la calidad y la cantidad de datos que respaldan su utilización, la investigación sobre MTC debería utilizar los métodos generalmente aceptados para evaluar los servicios de salud, en particular los estudios comparativos de la eficacia y los modelos de métodos combinados. Debemos de remarcar que la Medicina Tradicional y Complementaria debe de basarse necesariamente en evidencia científica, para que pueda ingresar formalmente en el sistema de atención sanitaria, esto se puede lograr con más esfuerzos de investigación e innovación y una atención centrada en la gestión de conocimientos, incluidos los derechos de protección de la propiedad intelectual, por tanto constituye un reto para la investigación científica y la generación de evidencia científica de la cual forma parte nuestra revista científica Ágora. 


\section{REFERENCIAS BIBLIOGRÁFICAS}

1. WHA62.13. Medicina tradicional. En: $62^{\mathrm{a}}$ Asamblea Mundial de la Salud, Ginebra, 18- 22 de mayo de 2009. Resoluciones y decisiones, anexos. Ginebra, Organización Mundial de la Salud, 2009 (WHA62/2009/REC/1;

http://apps.who.int/gb/ebwha/ pdf_files/WHA62-REC1/WHA62_REC1sp-P1.pdf.

2. Estrategia de la OMS sobre medicina tradicional: 2014-2023, OMS, Ginebra. WHO Western Pacific Regional Strategy on traditional medicine: 2011-2020. Manila, Oficina Regional de la OMS para el Pacífico Occidental, 2012. (ISBN 97892 9061559 0)

3. Estrategia de la OMS sobre medicina tradicional 2002-2005. Ginebra, Organización Mundial de la Salud, 2002 (WHO/EDM/TRM/2002.1).

4. WHO medicines strategy.2004-2007. Ginebra, Organización Mundial de la Salud, 2004 (WHO/EDM/2004.5).

5. Chao, S. et al. Musculoskeletal disorders: Does the osteopathic medical profession demonstrate its unique and distinctive characteristics? Journal of the American Osteopathic Association, 2004, 104(4):149-155.
6. Rossignol, M. et al. Who seeks primary care for musculoskeletal disorders with physicians prescribing homeopathic and other complementary medicine? Resultados de la encuesta EPI3-LASER en Francia. BioMed Central (BMC) Musculoskeletal Disorder, 2011, 12: 21-26. (http://www.biomedcentral.com/14712474/12/21)

7. Skovgaard, L. et al. Use of Complementary and Alternative Medicine among People with Multiple Sclerosis in the Nordic Countries. Autoimmune Diseases, 2012: 841085, Publicado en línea el 11 de diciembre de 2012. doi: 10.1155/2012/841085 (http:// pubmedcentralcanada.ca/pmcc/articles/PM C3529905/).

8. Zhang, Q. et al. The importance of traditional Chinese medicine services in health care provision in China. Universitas Forum, 2011, 2(2): 1-8.

Dr. Walter Gómez ${ }^{1}$

${ }^{1}$ Oficina de Investigación y Creatividad Intelectual - Universidad María Auxiliadora- Lima-Perú. Editor Jefe - AGORA Revista Científica. 